\title{
Industrial Plant Drawer ${ }^{\star}$
}

\author{
Walter Didimo $^{1}$, Maurizio Patrignani ${ }^{2}$, and Maurizio Pizzonia ${ }^{2}$ \\ 1 Università di Perugia, Perugia, Italy \\ 2 Università di Roma Tre, Rome, Italy
}

\section{Short Description}

Industrial Plant Drawer is a prototypical system especially designed to cope with the complexity of the requirements of industrial plant schemas. It produces orthogonal drawings in which the size of the nodes is assigned according to the symbol associated with them. Also, it allows the user to choose a set of nodes to be placed on the border of the drawing. Labels, colors, and other graphic features are dealt with. Created as a GDToolkit demo, Industrial Plan Drawer grew into an independent system.

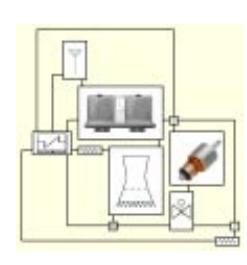

(a)

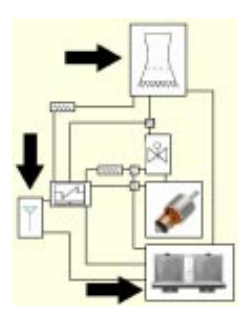

(b)
Fig. 1. Two drawings generated with Industrial Plant Drawer. In (a) some of the vertices are replaced with symbols of appropriate dimensions. In (b) the same graph where the highlighted nodes are constrained to be on the border.

\section{Areas of Application}

Engineers who design, maintain, and manage industrial plants extensively use diagrammatic representations which are necessary to understand the complexity of the industrial systems they deal with. In such schemas each node represents a component of the plant, and each edge represents flow of material or control information. They are usually very large, difficult to modify or update, and produced by hand in the design phase of the life-cycle of the plant.

\section{Layout Algorithms and Layout Features}

Even though industrial plants may be represented as graphs, they are particularly challenging for the currently available graph drawing tools. One of the features that most affect the readability of industrial schemas is concerned with the size of each symbol that should reflect the role it plays in the system or its real dimensions. Also, devices that interface the system with other systems

\footnotetext{
* Work partially supported by: "Progetto Algoritmi per Grandi Insiemi di Dati: Scienza e Ingegneria”, MURST Programmi di Ricerca di Rilevante Interesse Nazionale. 


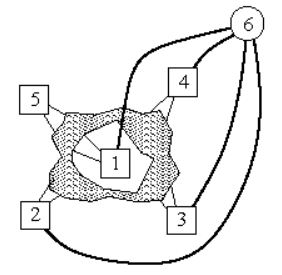

(a)

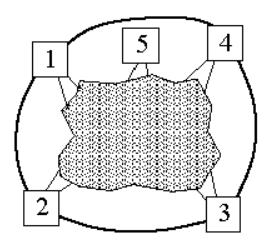

(b)

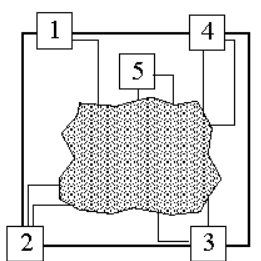

(c)

Fig. 2. This figure illustrates the procedure that our system uses to constrain some nodes $(1,2,3$, and 4 in the picture) on the boundary of the drawing.

should be placed on the boundary of the drawing (see Figure 1). Orthogonal drawings with prescribed vertex size are produced with a topology-shape-metrics approach, similar to the one described in [1]. Since some nodes need to be placed on the border of the drawing, a dummy node is connected to them with uncrossable edges (see Figure 2(a)). After planarization the dummy node is removed and the constrained nodes are connected in a cycle as in Figure 2(b). Orthogonalization is then performed with the constraint that walking on such cycle counterclockwise its edges can only turn to the left. This forces the shape of the external face to be a rectangle (see Figure 2(c)). Then compaction is performed as described in [1]. The edges of the rectangle are removed before visualization.

Industrial Plant Drawer is written in $\mathrm{C}++$ language, using the GDToolkit graph drawing library [2] and LEDA [3], and runs on all the operating systems supported by these libraries.

\section{Interfaces}

Industrial Plant Drawer is extremely friendly. A graph is inserted through the graph editor or by loading it from a file. The user can customize the drawing through dialog boxes that pop up by clicking on the nodes and edges. Each node can be associated with a plant component chosen from a collection. Furthermore, the user can specify whether the node should be constrained to be on the boundary of the drawing. Each edge may be directed, colored, labeled, etc.

\section{References}

1. G. Di Battista, W. Didimo, M. Patrignani, and M. Pizzonia. Orthogonal and quasiupward drawings with vertices of prescribed size. In J. Kratochvil, editor, Graph Drawing (Proc. GD '99), volume 1731 of Lecture Notes Comput. Sci. SpringerVerlag, 1999.

2. Gdtoolkit 3.0: An object-oriented library for handling and drawing graphs, 1999. Third University of Rome, http://www.dia.uniroma3.it/ gdt.

3. K. Mehlhorn and S. Näher. LEDA: A Platform for Combinatorial and Geometric Computing. Cambridge University Press, New York, 1998. 